\title{
Signal Feature Extraction for Sewing Analysis using Non-Linear Techniques
}

\author{
H. CARVALHO ${ }^{1}$, Member, IEEE, P. FERREIRA ${ }^{2}$, A.ROCHA ${ }^{1}$ and J..MONTEIRO ${ }^{2}$, Member, IEEE \\ ${ }^{1}$ UM, DET, Campus de Azurém, Guimarães, Portugal, e-mail (helder@det.uminho.pt, amrocha@det.uminho.pt) \\ ${ }^{2} \mathrm{UM}, \mathrm{DEI}$, Campus de Azurém, Guimarães, Portugal, e-mail (pedro_celestino@sapo.pt, monteiro@dei.uminho.pt)
}

\begin{abstract}
This paper describes the measurement of a process variable in industrial sewing machines using a piezoelectric sensing system and non-linear filtering and processing techniques. The objective is to obtain a measure of the needle penetration and withdrawal forces in a fabric, which can be used to detect faulty processing conditions causing second-rate quality products. Aimed for future real-time applications, currently the measurement process has been optimised for laboratory use. It provides the means for experimentation leading to the development of further processing techniques able to detect fault conditions automatically, a completely innovative industrial application.

The signal is acquired by a piezoelectric sensor introduced in the needle support bar. With this set-up, the sensor provides not only the forces related to needle-fabric interaction, but also other, undesired components, that are reduced with specifically developed techniques. This paper presents the advantages and drawbacks of the measurement set-up and describes the optimization of the complex processing techniques used to extract the relevant information from the signals.
\end{abstract}

Index Terms-sewing, monitoring, sensors, filtering, neural processing

\section{INTRODUCTION}

Although a great range of mechanical and electronic technologies has boosted the development of industrial high-speed sewing machines, the process itself, in which threads are interlaced in or around fabrics at high speed, is a very complex one, and there is little quantitative knowledge about it.

This knowledge is important to serve as a basis for the development of devices that can monitor and in some cases control the process variables, and for the development of tools for an up-front engineering of the process and test of the materials. This better control of the process can increase quality, reduce set-up times (thus improving flexibility) and reduce stops due to technical problems that in industrial practice often go unnoticed for a significant amount of time, resulting in the production of defective products.

Several researchers have developed sewing test rigs to study this subject [1][2][3]. At the University of Minho, research in this area has been developed since 1988. The sewing process has been observed in an integrated way, although process variables can to a certain degree be studied separately. Basically, three different groups of process variables of the machine can be distinguished:
- Related to stitch formation: Thread tensions and thread consumption during sewing;

- Related to material feeding: Force on and displacement of the presser-foot (the component that presses the fabric against the feeding device);

- Related to the penetration of the needle: Force on the bar that holds the needle.

The studies performed to this point have already provided a significant insight into the mechanics of the sewing process. These insights have been materialised into a software application that is able to analyse and supply a great amount of indicators describing the efficiency of the process. These indicators can be used to monitor the operation of the sewing machine and provide feedback variables for closed-loop control systems (for some of the subsystems of the machine) [5][6].

The most difficult measurement to be taken is that of the needle-fabric interaction. The incorporation of a piezoelectric sensor into the needle-bar supplies a signal in which several effects are present, of which only the needle-fabric interaction is of interest to the analysis. Although much information has already been obtained studying the original signal qualitatively and using simple processing techniques, a fully automatic and more accurate feature extraction method is seeked for. This paper describes the optimization of this method.

\section{The Measurement Set-UP}

This sewing test rig (Fig.1) is built around an industrial overlock machine and is essentially composed of the following elements:

- Sensors to convert several of the processes' variables:

- Semiconductor strain gauges in cantilever beams for thread tensions, digital encoders counting thread consumption;

- Force on presser-foot and needle-bar with piezoelectric transducers;

- A LVDT for presser-foot displacement;

- Conditioning Hardware;

- A PC equipped with a data acquisition board;

- Software. 


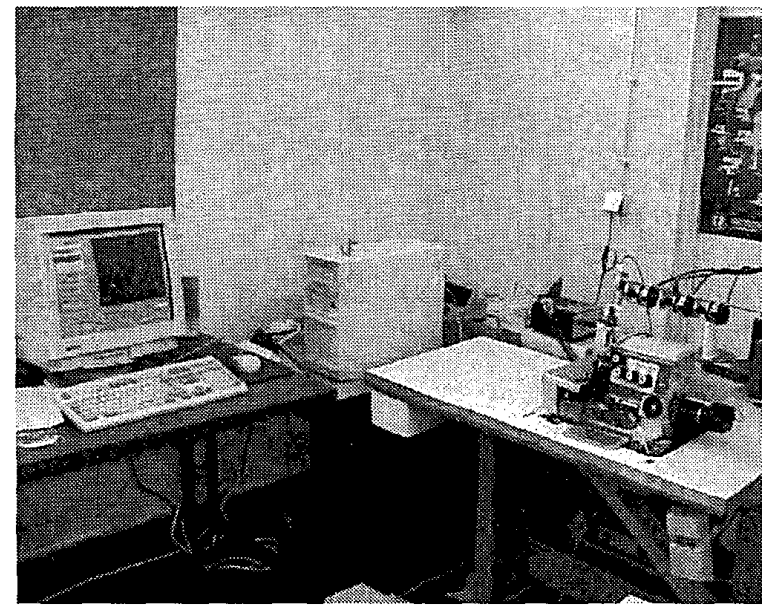

Fig. 1: Overview of the sewing test rig

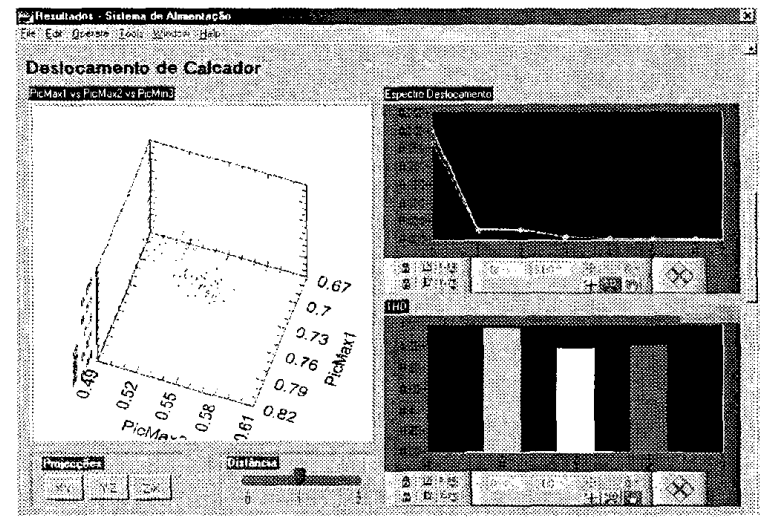

Fig.2: Screenshot of the software, in the example showing information about the performance of the feeding system

The software, developed with LabView, is the keystone of the research work performed and has been in development for several years. In addition to basic functions like acquisition, file I/O, graphical display and sensor calibration, an extensive set of specific analysis tools have been progressively added, of which the processing of needle-bar force signal is part.

Needle-bar force is measured with a sensor introduced into the needle-bar, as shown in Figure 3.

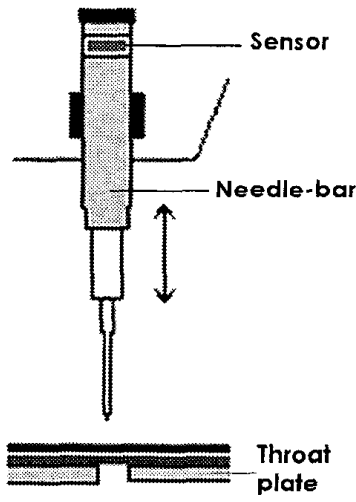

Fig.3: Sensor set-up
The position of the sensor on the top of the needle-bar creates the disadvantage of adding the acceleration forces of the whole needle-bar mass to the measurement, although only the interaction between the needle and the fabric is relevant to the objectives of the research undertaken. Placing the sensor in the lower part of the needle-bar would reduce the acceleration components significantly. This is mechanically not possible on the machine used, but it can be done on other types of machines and also on this type upon redesign of the needle-bar arrangement.

In actual sewing, a third component is also present in the signal, produced by the thread forces (the needle carries a thread through the fabric). For the purpose of laboratorial analysis, this component can be eliminated by unthreading the needle. The presence of this new component is another effect blurring the measurement, but it has been found that the system is still able to distinguish different sewing conditions with the presence of the thread. Needle penetration forces, when relevant to the analysis, are much higher than the thread forces.

Although presenting the inconvenience of producing undesired components on the measured signal, this set-up is the only possibility of capturing all the effects of needlefabric interaction. An alternative would be the use of sensors in the machine's throat plate, which provides an indirect measurement of needle penetration force, but not the important withdrawal counterpart. Commercial sewability testers providing measurement of needle penetration are designed as laboratory instruments the sensor is not integrated in an actual sewing machine. They are also unable to perform the measurement at high sewing speeds.

Figure 4 shows the comparison of a signal acquired without fabric (no needle-fabric interaction present, a void signal) and that obtained stitching two plies of a denim fabric at about 1000 stitches per minute (spm).

As can be seen, the signal content produced by the needle-fabric interaction are bursts produced in the phase of the sewing cycle in which the needle touches, penetrates and withdraws from the fabric.

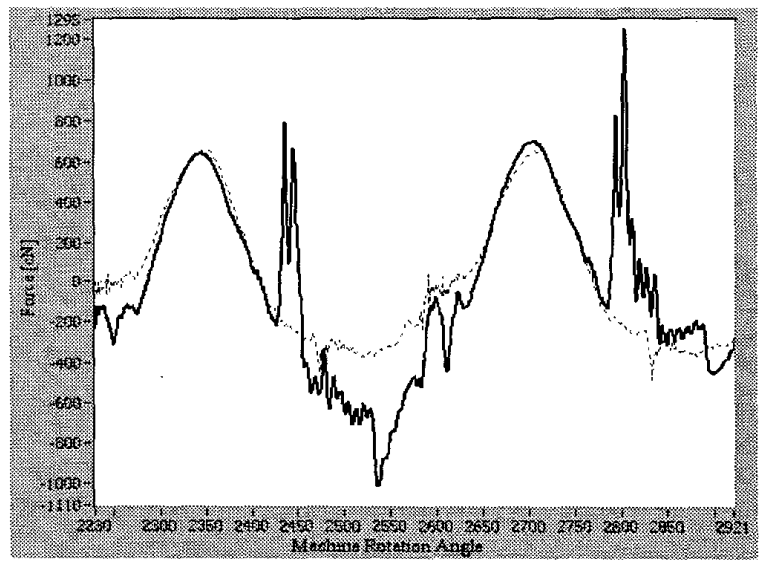

Fig 4: Needle-bar force without fabric (dashed) and with 2 ply of a denim fabric, at 1000 stitches per minute (solid) 
In the case presented, the thick denim fabric used produces significant penetration and withdrawal forces. In addition, the signal has been acquired at 1000 stitches per minute, a low speed. At higher speeds and with thinner materials, signal-to-noise ratios are much lower, although for most situations worthy of study, the needle still produces a measurable effect.

\section{FIRST APPROACHES TO SIGNAL PRE-PROCESSING}

The most sensitive method for eliminating the acceleration force component is to acquire a void signal at the same sewing speed as the original signal and perform a simple subtraction. This method has several restrictions that render it inadequate for a real-time application, and also somewhat limits its use for research purposes:

- Although time-scale can be corrected for speed differences, the acceleration force signal should be acquired at the same sewing speed as the signal it is subtracted from, in order to also avoid amplitude differences;

- The method is only applicable to seams produced at constant speed ;

- Differences in the offset of the two signals may produce errors;

- This method assumes that the motional forces are as stationary in the presence of fabric as they are when the acquisition is performed without fabric, which is not true .

However, this approach may be used in controlled conditions. Although the shape of needle penetration signals vary from stitch to stitch, the example depicted in Fig.5, obtained with the subtraction method, shows all events expected to happen during a whole needle penetration cycle. Three distinct phases are observed:

- Phase 1: First contact of needle tip with the fabric;

- Phase 2: The needle shaft penetrates, producing a second peak:

- Phase 3: The needle-bar inverts its movement and the fabric holds the needle, producing a third, inverted force peak (a valley).

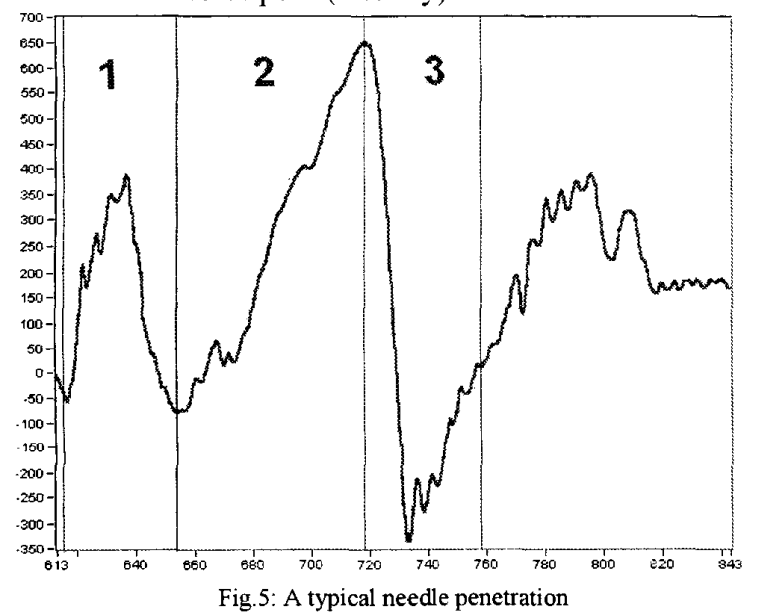

After pre-processing, several parameters are calculated on the signal subsets extracted from the signal according to the three penetration phases defined. The most important and most used are the peaks in phase 1 and 2, valleys in phase 3 and power in all of the three signal phases. The values of these features and their interrelations are used in the textile studies to evaluate the efficiency of needle penetration.

\section{SIGNAL PRE-PROCESSING USING FFT-BASED FILTERING}

Although the spectral analysis of signals acquired with and without fabric had shown that the penetration effect and the motion forces intersect in the frequency domain, both effects appeared sufficiently separated to allow the use of digital filtering techniques. In an attempt to implement as closely as possible an ideal filter, and to enable a flexible choice of its stopband, an FFT-based spectral filtering and reconstruction method was implemented, as shown in Fig.6.

The choice of the frequency components to eliminate from the spectrum has to be a compromise between the following two conflicting objectives:

- Attenuating the acceleration force component as much as possible;

- Causing as low distortion to the needle penetration component as possible.

A first version of the filtering algorithm has already revealed that the measurement system is able to clearly mark out different sewing conditions (different number of fabric plies, needle condition, fabric type and finishing, etc.) The objective is now to optimize the measurement and advance to a new series of tests aiming to describe the behavior of forces related to sewing conditions in a quantitative way.

To perform this optimization, the spectrum of signals acquired at low, medium and high-speed situations have been observed to describe the signal's spectral content. It was found that the most important components present in the signal are the fundamental, and the second to third harmonics. With respect to the elimination of acceleration forces, these would be the harmonics to be eliminated.

The second step was to determine the distortion caused by the filtering process on the penetration signal. But this study is not possible to be performed on real signals, as it is possible to obtain acceleration signals without needle-fabric interaction, but not the opposite.

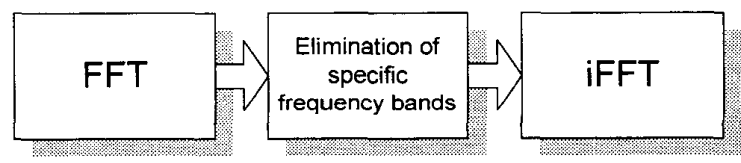

Fig.6: Principle of FFT-based filtering 


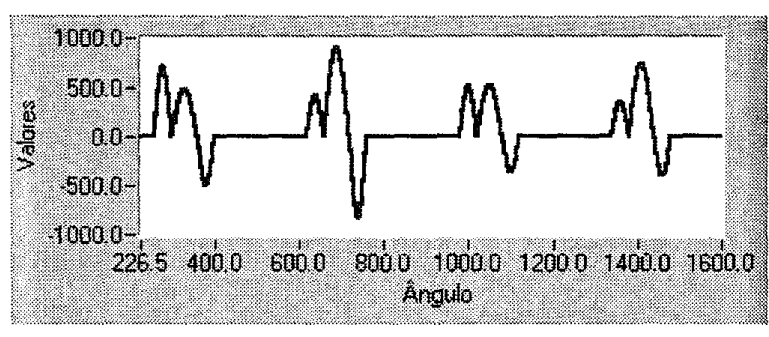

Fig.7: Simulated penetration signal based on one of the developed models

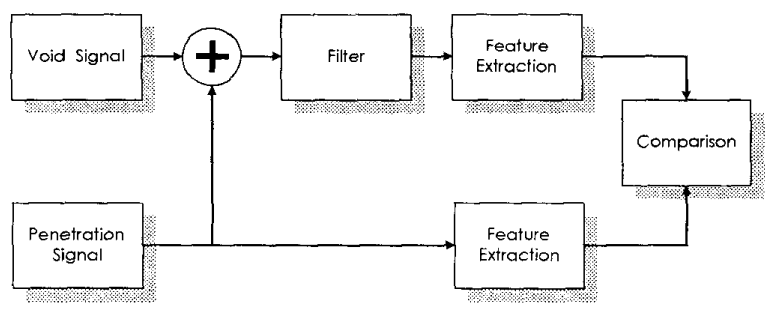

Fig. 8: Filtering optimization using simulated penetration signals

To overcome this problem, a program was developed that generates simulated penetration signals based on various models derived from the observation of typical needlebar force signals. These are composed of three half-periods of a sine wave, with the typical duration of the penetration phases and amplitude that may be constant, random, or a combination of a constant base value and an added random value (Fig.7).

The program simulates the whole filtering and feature extraction procedure, comparing the feature values extracted from the original signals (the correct values) with those obtained from the filtered signal (Fig.8). The error produced by the filtering procedure can thus be quantified and the pre-processing method optimized.

Quantification of the distortion produced by filtering has been obtained using the following parameters:

- Correlation between correct feature values and those obtained after the filtering process;

- Average relative error produced;

- Standard deviation of the relative error as a measure of error variability.

A preliminary study using this software tool showed that the distortion of the simulated penetration signals varies with the relation of amplitude between the three half-sines. This is an important observation, since in real penetration signals these amplitude ratios may also vary with the type of fabric being stitched. To include this factor in the optimization, the program was modified to generate sets of signals representing various amplitude relations, in a number of selectable levels of the base-amplitude indicated.

The study was then performed considering different stopbands that are selective combinations of the DC, fundamental component and the first three harmonics. The elimination of the DC component eliminates offset fluctuations due to the high-pass characteristic of the piezoelectric measurement system, and can be compensated with zero correction, a method described in [4].

The study was then carried out in two stages. In the first one, void signals acquired at different speeds were processed and the ability of the filter to eliminate the mechanical noise was analyzed. Specifically, this method observes the feature values (peak and power of the signal subsets extracted in the three needle penetration phases) obtained when processing void signals - signals in which no penetration occurs and that should consequently result in values as close to zero as possible. Fig. 9 shows the peak values extracted from void signals in phase 1 , as an example.

After analysis of peak and power values in the three phases, it was found that the $0-1-3 C$ or $0-1-2-3-C$ stopbands show the best results in the elimination of the mechanical noise for the purposes of the measurement. ("0-1-3C" stopband: Elimination of dc, fundamental and third harmonic, with subsequent zero correction).

The results of this stage of the study provide an additional valuable information: They show the noise floor associated with this measurement, providing a means of deciding whether a penetration force value obtained is an actual effect of needle penetration or just noise.

In the second stage of the study, a set of simulated penetration signals with varying amplitude combinations was processed to determine in what extent they are distorted using the different stopbands. The most significant result is depicted in the chart of Fig.10, that shows the average correlation between the correct and measured peak values in the three phases.

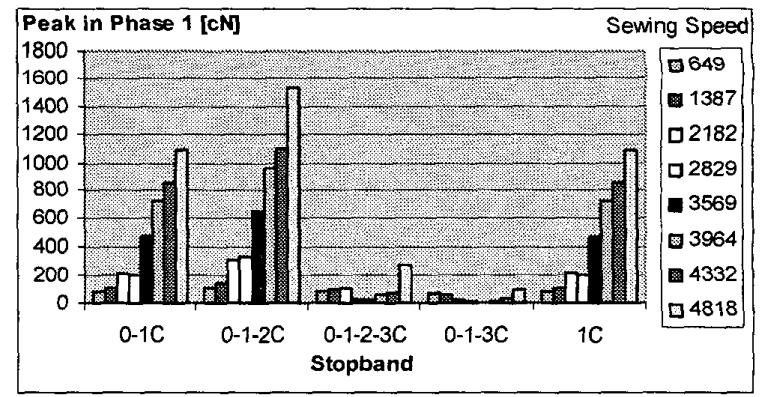

Fig 9: Peak values extracted from void signals in phase 1 using different stopbands

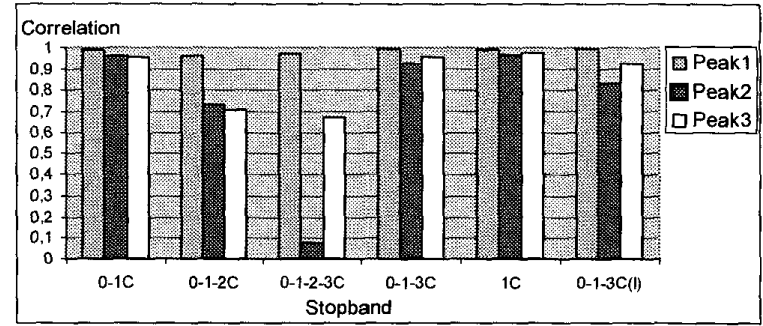

Fig.10: Average correlation between correct and measured values of peaks in the three phases, using different stopbands 
The examination of these two charts and the remaining results, not shown here, has led to the choice of the $0-1-3 \mathrm{C}$ stopband as the best compromise to fulfil the objectives stated earlier. This choice was then validated in a third stage, in which void signals were finally mixed with simulated penetration signals and the same analysis was performed.

The results obtained regarding errors, however, showed that the measurement process is inaccurate, providing only an estimate of the feature values. The average relative error of approx. $-40 \%$ reflects the attenuation produced by the filter and can be accounted for, but this error is highly variable form stitch to stitch, with a standard deviation of 20 to $30 \%$. An attempt to improve performance was made by using neural networks, as described in the next section.

\section{VALUE CORRECTION USING NEURAL NETWORKS}

To improve the accuracy of the measurement process, a neural network was trained approximate the following function:

$$
\left[y_{1} ; y_{2} ; y_{3}\right]=f\left(x_{1}, x_{2}, x_{3}\right)
$$

$$
\begin{aligned}
\text { where } & \\
y_{n}: & \text { Original feature value for phase } n \\
x_{n}: & \text { Feature value extracted from signal in phase } \\
& n \text {, after filtering }
\end{aligned}
$$

The goal is to filter the signal, extract the feature values and correct them using the Neural Network.

A Multilayer Perceptron (MLP) structure with one hidden layer was trained with data sets generated by the simulation program (the networks were implemented with Matlab). Two training sets, one for a network to be trained for peaks/valley correction, and another for power values, were generated considering the following variables:

- Varying amplitude relations between the three phases in the penetration signal;

- Void signals at three different sewing speeds;

- Varying signal-to-noise ratios for each sewing speed:

- One penetration signal model;

To account for varying acceleration force amplitude and spectral content, a $4^{\text {th }}$ variable, sewing speed, was added to equation 1.

The performance of the network has been found to be very good when trained and tested with simple data sets, all generated with the same shape parameters (same amplitude ratio, sewing speed, signal-to-noise ratio).

When using the more general training sets in which all of the conditions are varied, the network is still able to converge well on the training set for peak value correction. But when tested with an independently generated test set, the accuracy of the result is not very different from the ones obtained directly after filtering.

In general, correlation appears slightly higher, but the error variability, one of the parameters most important to be improved, does not reflect this change. In lower signal-tonoise ratios, the values obtained with the neural network are slightly worse than the values after filtering. Average error is in general lower, but not consistently in all sewing speeds. These observations lead to the conclusion that the neural network merely produces an offset shift and scaling to the values.

Although it is not clear if the peaks/valley correction provided by the network is useful, it has been integrated into the sewing test rig's software as an alternative analysis method.

In the course of the research project, several experiments have been carried out and results using the neural correction have been compared to their uncorrected counterparts. Situations with predictable results have been produced to determine if the neural-corrected values are able to uncover any effects that are not found with the simple filtering procedure, but no such improvement has yet been found.

As an example, samples of a jersey knitwear have been stitched at $1000 \mathrm{spm}$ using a very thick needle $(1.2 \mathrm{~mm}$ diameter), that produced damage to the fabric in some situations. The fabric has been used in three states of finishing: raw, dyed and calendered, and finished with thermofixing and softening.

Regarding the defect situations, it has still not been possible to find any sign that would enable the detection of damage neither with the corrected nor with the uncorrected values.

The measurement system is, however, perfectly able to distinguish the three different states of finishing. Figs. 11 and 12 show the results of peaks/valleys in phases 1,2 and 3 , plotted on a 3-D graph, a new tool developed to observe the results of needle penetration forces. Fig.11 shows the uncorrected, whilst Fig.12 shows the uncorrected values.

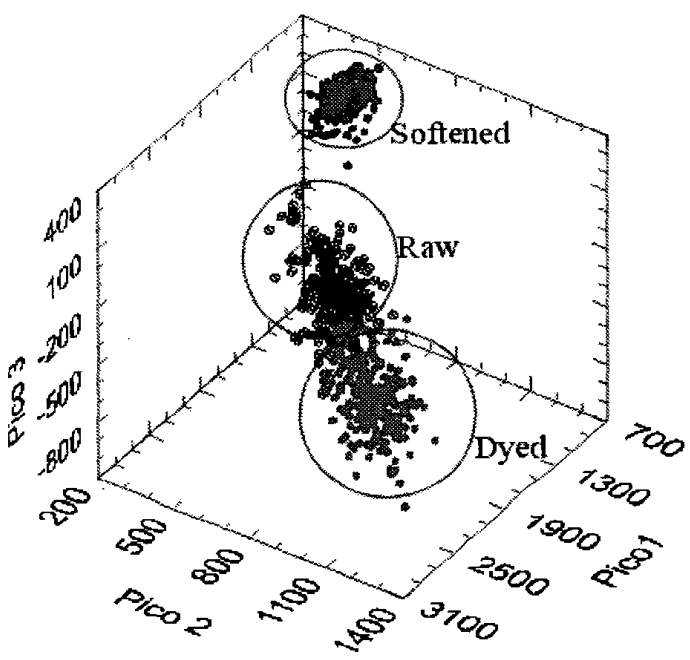

Fig.11: Uncorrected peak values for raw, dyed and softened fabric 


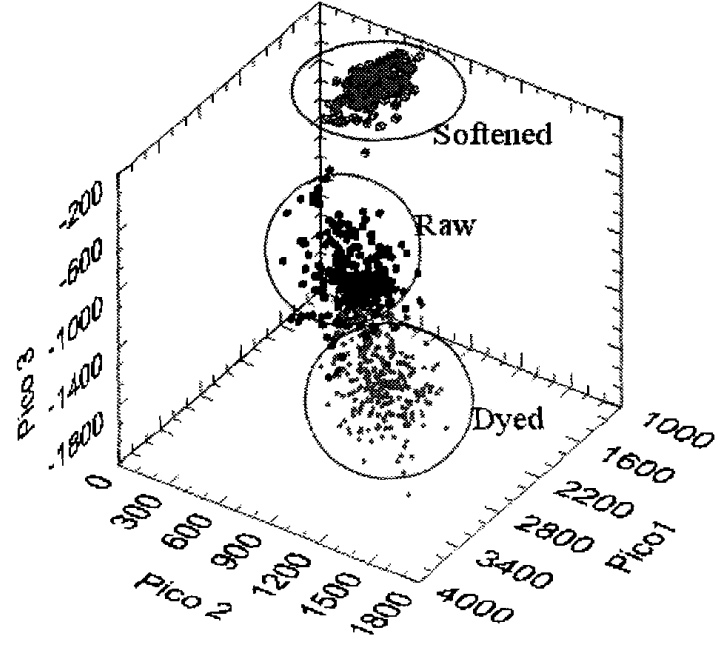

Fig.12: Corrected peak values for raw, dyed and softened fabric

As can be observed, the values are qualitatively not different, but seem to be offset and/or scaled by the neural network. The corrected result seems more acceptable in some cases, as for instant Valley3, that appears with negative values in all cases of the corrected set. But the shift produced by the network is not consistent throughout all the situations produced: the same samples sewn at a different speed appeared shifted in a less correct way.

Nevertheless, data is still being analysed with and without this correction scheme; quantitatively the results seem not to be consistent, but there is still the prospect that in some situation the corrected values may reveal a qualitative characteristic not visible on the uncorrected data.

\section{Vi. Conclusions}

Due to the mechanical set-up of the sensor, the measurement of needle penetration signals becomes quite difficult. The processing techniques developed, although optimised as widely as possible, can only provide an estimate of the real values. Extensive practical experimentation under controlled sewing conditions is being undertaken to show if this estimation is sufficient for the objectives in hand.

First tests have shown that the system is able to show a clear distinction between different sewing situations, but fine differences are still invisible with the data produced so far.

An alternative mechanical set-up of the sensor and the reduction of the needle-bar's mass can reduce the parasitic components and thus improve measurement accuracy.
This set-up is being designed for another machine, and is theoretically possible in any machine if the needle-bar mechanics is slightly redesigned. The processing algorithms presented will in any case play a fundamental role in the evaluation of the needle penetration efficiency.

At the current state of development, and considering also the capabilities of the system in the evaluation of the other two subsystems of the machine, stitch formation and feeding system [5][7], the system is developing as a complete sewability tester. The results obtained using this equipment are being translated in specific applications of real-time monitoring and control of sewing machines[6], whilst the system is evolving both as a development tool and as an off-line material test and process planning instrument.

\section{REFERENCES}

[1] R. Chmielowiek, D. W Lloyd, "The Measurement of Dynamic Effects in Commercial Sewing Machines". Proceedings of The 3rdAsian Textile Conference, Vol. II, pp. 814-828, 1995

[2] H Carvalho, J. L. Monteiro, F.N. Ferreira; "Measurements and Feature Extraction in High-Speed Sewing"; Proceedings of the ISIE'97 (IEEE International Synposium on Industrial Electronics), Vol.3, pp 961-966; Universidade do Minho, Guimarães, 7-11 July 1997

[3] H. Carvalho, Medição e Análise de Parâmetros em Máquina de Costura Industrial, MSc Thesis, University of Minho, Portugal, 1998

[4] H. Carvalho, J.L. Monteiro, F.N. Ferreira, A. Rocha; "A Sewing Rig with Automatic Feature Extraction"; Proceedings of the 6th UK Mechatronics Forum International Conference (Mechatronics'98), pp 727-732, Skovde, 9 a 11 de Setembro 1998

[5] H. Carvalho, A. Rocha, J.L. Monteiro, "Measurement and Processing of Sewing Variables towards Real-Time Control and Offline Process Planning", Proceedings of the International Conference on Information Processing and Management of Uncertainty in Knowledge-Based Systems, ISBN 2-9516453-5-X (CD-ROM), Annecy, France, 1-5 July 2002

[6] L. F. Silva, M. Lima, F.N. Ferreira, A. Rocha, H. Carvalho, C.Couto; "A New Electromagnetic Actuated Fabric Feeding System for Sewing Machines"; Proceedings of the 7th Mechatronics Forum International Conference - Mechatronics2000, Pergamon Press, ISBN 0080437036 (CD-ROM), Georgia Institute of Technology, Atlanta, USA, 6-8 Setembro 2000

[7] H.Carvalho, A.Rocha, J.L.Monteiro, "Process Control in NextGeneration Sewing Machines: A Project Overview", accepted for presentation and publication at the International Conference on Mechatronics (ICOM 2003), 19-20 $0^{\text {th }}$ June 2003, Loughborough University, UK

\section{ACKNOWLEDGEMENTS}

The authors are grateful to FCT (Fundação para a Ciência e Tecnologia), project funding POSI/SRI/38944/2001. 